Gut, 1979, 20, 660-665

\title{
Significance of tumour mass on t-lymphocyte levels in patients with gastrointestinal cancer
}

\author{
H. S. SHUKLA ${ }^{1}$, R. H. WHITEHEAD ${ }^{2}$, AND L. E. HUGHES \\ From the University Department of Surgery, Welsh National School of Medicine, Cardiff
}

SUMMARY The relationship between tumour load and immunity in gastrointestinal cancer has been studied by sequential comparison in patients whose tumour has been removed and those whose tumour was found to be inoperable. Total lymphocyte count, absolute and percentage T- and B-lymphocyte counts, effect of papain on E-rosetting cell levels, and inhibitory effect of cancer sera on E-rosette formation by normal lymphocytes have been studied in 30 patients with stomach or colorectal cancer, and 10 control patients with benign gastrointestinal disease. The examination was done on each patient before and at regular intervals after operation up to 24 weeks. Operable cases, with removal of tumour load, showed a temporary fall in total lymphocyte count and $\mathrm{T}$ cell counts, which returned to normal by four weeks postoperatively. Inoperable cases (15 patients) showed a progressive fall in total lymphocyte count and a relatively greater depression of $T$ cell counts, in parallel with increasing tumour mass. E-receptor blocking factor was demonstrated in the sera of cancer patients. This factor was related to tumour mass and presumably was of tumour origin, as it persisted in the inoperable group but disappeared by 12 weeks after tumour removal. The factor explained the excess depression of $T$ cells over total lymphocytes, but does not explain the continuing depression of total lymphocyte count in the cancer patients.

Studies of lymphocyte subpopulations in cancer patients have shown a decrease in T-lymphocyte population with corresponding increase in the null (non-rosetting) cells-a finding correlating with other evidence of depressed cellular immunity in similar patients (Bolton et al., 1975). The nature of T-lymphocyte depression has been explored but reports on the nature of the factor responsible are contradictory. Thus Al-Sarraf et al. (1971) postulated that impaired in vitro responsiveness of lymphocytes to PHA was due to an intrinsic cellular abnormality, whereas other workers have reported that impaired lymphocyte function was due to an inhibitory factor present in the serum (Sample et al., 1974). Others (Golob et al., 1969) have not found cancer sera inhibitory to normal lymphocytes.

In breast cancer patients, Whitehead et al. (1976) found that lowered percentage of T-lymphocytes in breast cancer returned to normal after treatment with papain, but fell again after their resuspension

${ }^{1}$ Present address: MLN Medical College, University of Allahabad, Allahabad, India.

'Present address: Peter McCallum Institute, 481 Little Lonsdale Street, Melbourne, Victoria, Australia.

Received for publication 29 March 1979 in patients' serum, indicating the presence of an E-receptor masking factor in the sera. In view of the role of T-lymphocytes in immune surveillance, the persistence of such a serum factor after treatment would continue to depress 'functional' T-lymphocyte levels and might contribute to the spread of cancer. If the serum factor is a tumour-related product, it should disappear from the serum after successful treatment. We have therefore investigated the effect of tumour load on a number of immune parameters, by comparing sequential investigations in two groups of patients with gastrointestinal cancer, one operable-with tumour load returning to a minimal state - and one inoperable with progressive increase in tumour mass.

\section{Methods}

Forty patients were studied: 10 healthy controls with benign gastrointestinal conditions other than inflammatory bowel disease (aged 50-77 years; mean 64 years); 15 patients with either carcinoma of the stomach (four), colon (seven), or rectum (four) with apparently localised cancer removed by surgery (aged 52-78 years; mean 63 years); and 15 cases of either carcinoma of the stomach (11), colon (one), 
or rectum (three) found to be inoperable at laparotomy because of visceral metastases (aged 56-74 years; mean 65 years). Total lymphocyte count, percentage E- and EAC-rosetting cells, and the effect of patients' serum on E-rosette formation of normal lymphocytes were studied. From these figures, absolute T- and B-lymphocyte counts and null cell counts were calculated. Examination was made before and two, four, eight, 12, and 24 weeks after operation. No patient was on drugs affecting the immune mechanism and, when an alternative treatment was started (chemotherapy or radiotherapy), the study was discontinued for that patient.

\section{ROSETTING METHOD}

Fifteen millilitres of blood were taken from a peripheral vein and the lymphocytes separated on Ficoll/Hypaque. The lymphocytes were washed twice in normal saline solution and $\mathrm{T}$ - and $\mathrm{B}$ lymphocytes were determined using E- and EACrosetting techniques as described by Anthony et al. (1975). Both lymphocytes and sheep erythrocytes were suspended in isotonic saline and were incubated in isotonic saline during the test. A routine full blood count was done for total lymphocyte count and for derivation of absolute T- and B-lymphocyte counts.

\section{PAPAIN TREATMENT}

The lymphocytes were separated and washed as before and were then incubated with $0.66 \mathrm{mg} / \mathrm{ml}$ papain (twice crystallised, 11 units/mg, Sigma, London) in $2.5 \mathrm{mmol} / 1$ cysteine hydrochloride in Earle's balanced solution containing $5 \%$ human serum for one hour at $37^{\circ} \mathrm{C}$ (Chapel, 1973). After incubation the lymphocytes were washed once and rosetting was done as described.

\section{SERUM INHIBITION}

The lymphocytes of normal healthy controls were incubated with the serum of patients at $37^{\circ}$ for one hour. After the incubation, lymphocytes were washed twice in isotonic saline and E-rosetting carried out. From the E-rosette percentage after incubation in autologous serum or cancer serum, percentage inhibition of normal T-lymphocytes was calculated (Whitehead et al., 1977).

\section{VIABILITY}

The viability of lymphocytes was checked using dye exclusion methods.

Table 1 Gastrointestinal cancer: E-rosette formation and absolute $T$ cell count (all results expressed as mean +1 SD)

\begin{tabular}{|c|c|c|c|c|c|c|c|}
\hline & & \multirow[t]{2}{*}{ Pretreatment } & \multicolumn{5}{|l|}{ Weeks } \\
\hline & & & 2 & 4 & 8 & 12 & 24 \\
\hline $\begin{array}{l}\text { Control } \\
\text { Operable cancer } \\
\text { Inoperable cancer }\end{array}$ & $\begin{array}{l}\text { E rosette (\%) } \\
\text { Absolute T-lymphocyte count } \\
\text { E rosette (\%) } \\
\text { Absolute T-lymphocyte count } \\
\text { E rosette (\%) } \\
\text { Absolute T-lymphocyte count }\end{array}$ & $\begin{array}{l}72+3 \\
1538 \\
66 \pm 10 \\
981 \pm 370 \\
56 \pm 11 \\
802 \pm 402\end{array}$ & $\begin{array}{l}- \\
- \\
56 \pm 9 \\
746 \pm 237 \\
49 \pm 13 \\
531 \pm 263\end{array}$ & $\begin{array}{l}- \\
\overline{62} \pm 11 \\
858 \pm 298 \\
51 \pm 15 \\
502 \pm 204\end{array}$ & $\begin{aligned} \overline{-} & \\
61 & \pm 14 \\
1144 & \pm 296 \\
43 & \pm 10 \\
449 & \pm 141\end{aligned}$ & $\begin{array}{l}- \\
-65 \pm 4 \\
965 \pm 173 \\
29 \pm 6 \\
259 \pm 125\end{array}$ & $\begin{array}{l}- \\
\overline{6} \\
66 \pm 2 \\
804 \pm 560 \\
25 \\
238\end{array}$ \\
\hline
\end{tabular}

Table 2 Gastrointestinal cancer: EAC-rosettes and absolute B cell counts (all results expressed as mean +1 SD)

\begin{tabular}{|c|c|c|c|c|c|c|c|}
\hline & & \multirow{2}{*}{ Pretreatment } & \multicolumn{5}{|l|}{ Weeks } \\
\hline & & & 2 & 4 & 8 & 12 & 24 \\
\hline & $\begin{array}{l}\text { EAC (\%) } \\
\text { Absolute B-lymphocytes }\end{array}$ & $\begin{array}{c}24 \pm 4 \\
590 \pm 231\end{array}$ & - & - & - & - & - \\
\hline $\begin{array}{l}\text { Operable cancer } \\
\text { Inoperable cancer }\end{array}$ & $\begin{array}{l}\text { EAC (\%) } \\
\text { Absolute B-lymphocytes } \\
\text { EAC (\%) } \\
\text { Absolute B-lymphocytes }\end{array}$ & $\begin{aligned} 24 & \pm 10 \\
383 & \pm 242 \\
28 & \pm 9 \\
388 & \pm 188\end{aligned}$ & $\begin{aligned} 29 & \pm 8 \\
341 & \pm 158 \\
26 & \pm 7 \\
263 & \pm 138\end{aligned}$ & $\begin{aligned} 31 & \pm 14 \\
341 & \pm 137 \\
27 & \pm 13 \\
275 & \pm 141\end{aligned}$ & $\begin{aligned} 22 & \pm 3 \\
372 & \pm 99 \\
30 & \pm 9 \\
300 & \pm 62\end{aligned}$ & $\begin{array}{c}26 \pm 9 \\
-32 \pm 6 \\
227 \pm 109\end{array}$ & $\begin{aligned} 30 & \pm 8 \\
424 & \pm 99 \\
42 & \pm 0 \\
390 & \pm 0\end{aligned}$ \\
\hline
\end{tabular}

Table 3 Gastrointestinal cancer: total lymphocyte count (all results expressed as mean \pm 1 SD)

\begin{tabular}{|c|c|c|c|c|c|c|}
\hline & \multirow[t]{2}{*}{ Pretreatment } & \multicolumn{5}{|l|}{ Weeks } \\
\hline & & 2 & 4 & 8 & 12 & 24 \\
\hline $\begin{array}{l}\text { Control } \\
\text { Operable cancer } \\
\text { Inoperable cancer }\end{array}$ & $\begin{array}{l}2213 \pm 891 \\
1467 \pm 524 \\
1440 \pm 716\end{array}$ & $\begin{array}{l}\overline{1357} \pm 515 \\
1084 \pm 524\end{array}$ & $\begin{array}{l}\overline{1291} \pm 359 \\
1024 \pm 538\end{array}$ & $\begin{array}{l}\overline{1737} \pm 317 \\
1044 \pm 288\end{array}$ & $\begin{array}{r}\overline{1135} \pm 622 \\
634 \pm 150\end{array}$ & $\begin{array}{r}\overline{1357} \pm 167 \\
952 \pm 108\end{array}$ \\
\hline
\end{tabular}




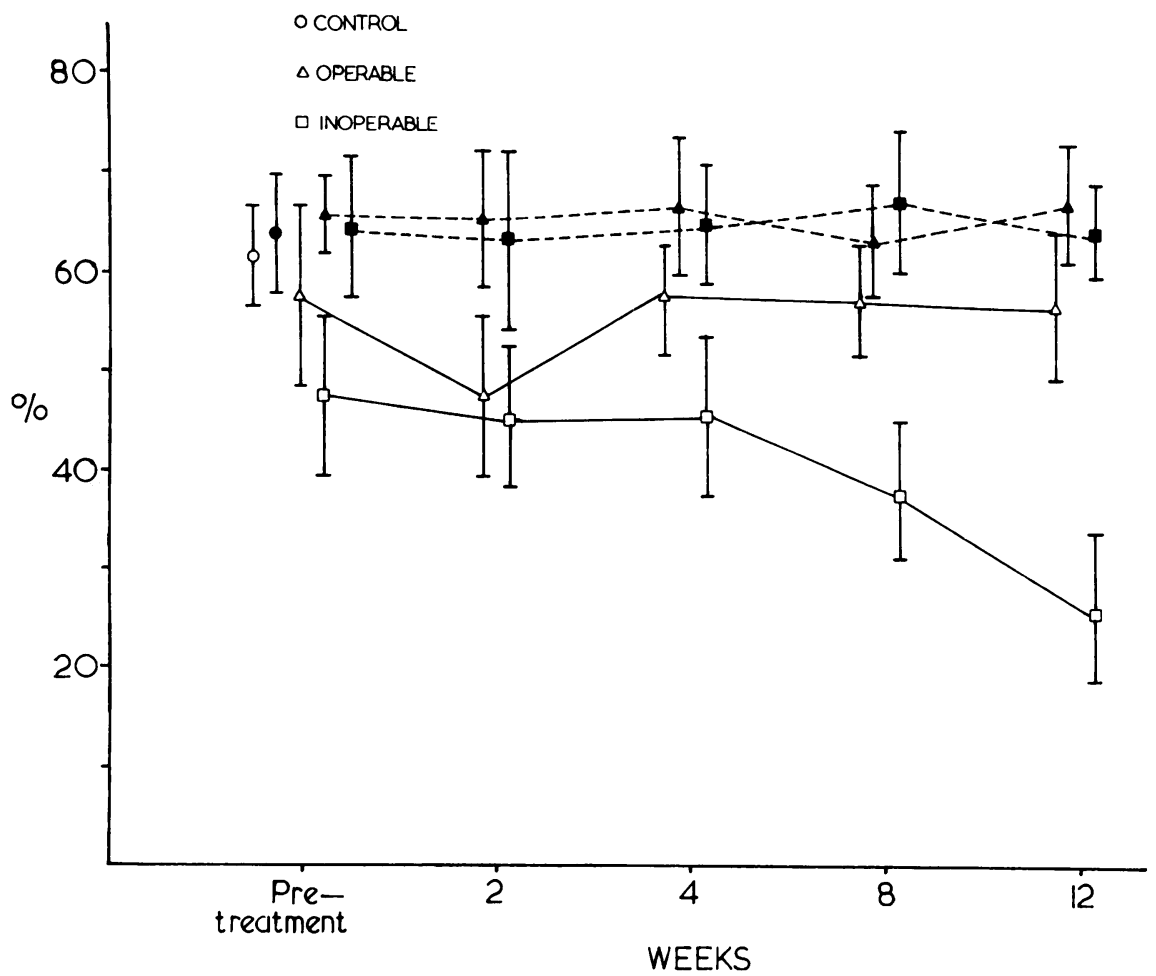

Fig. 1 Percentage E-rosetting cell levels before (open symbols) and after (closed symbols) papain treatment.

\section{Results}

\section{E-ROSETTE AND ABSOLUTE T-LYMPHOCYTE COUNTS}

The mean percentage of E-rosette and absolute $T$ cell counts for each group in the preoperative period and two, four, eight, 12, and 24 weeks after treatment is shown in Table 1. As compared with the controls, E-rosette and absolute $\mathrm{T}$ cell counts were depressed in cancer patients $(P<0.05$ and $<0.01$ respectively) but the depression in the inoperable stomach and colorectal cancer was significantly greater $(P<0.001)$ than in the operable group. After laparotomy the E-rosette and absolute $T$ cell counts continued to decrease $(P<0.001)$ in the inoperable group but in the operable group after an initial depression at two weeks $(\mathrm{P}<0.05)$ the levels recovered to preoperative values.

EAC-ROSETTE AND ABSOLUTE B CELL COUNTS These are shown in Table 2. There was no significant difference in the EAC percentage preoperatively or in the postoperative period in any of the group. However, the absolute $B$ cell counts were higher in the control group. The inoperable group showed a progressive fall in total B cell count that reflected the fall in the total lymphocyte count but did not reach a significant difference from the operable group.

TOTAL LYMPHOCYTE COUNT (Table 3)

The levels were significantly higher in controls $(P<0.05)$. Both operable and inoperable groups showed similar values in the preoperative period. After operation there was no change in the operable group but the levels in the inoperable group progressively diminished $(\mathrm{P}<0.01)$.

PAPAIN TREATMENT (Fig. 1)

Papain treatment increased the percentage of $\mathrm{E}$ rosetting cells in all the patients to levels similar to those of healthy control patients. This return of E-rosette percentage to normal was most marked in the inoperable group where, at 12 weeks postoperatively, the mean percentage T-lymphocyte levels increased from $29 \%$ to $74 \%$ after incubation in papain.

SERUM INHIBITION STUDIES

Figure 2 shows the inhibitory effect of the serum from controls and both the operable and inoperable 


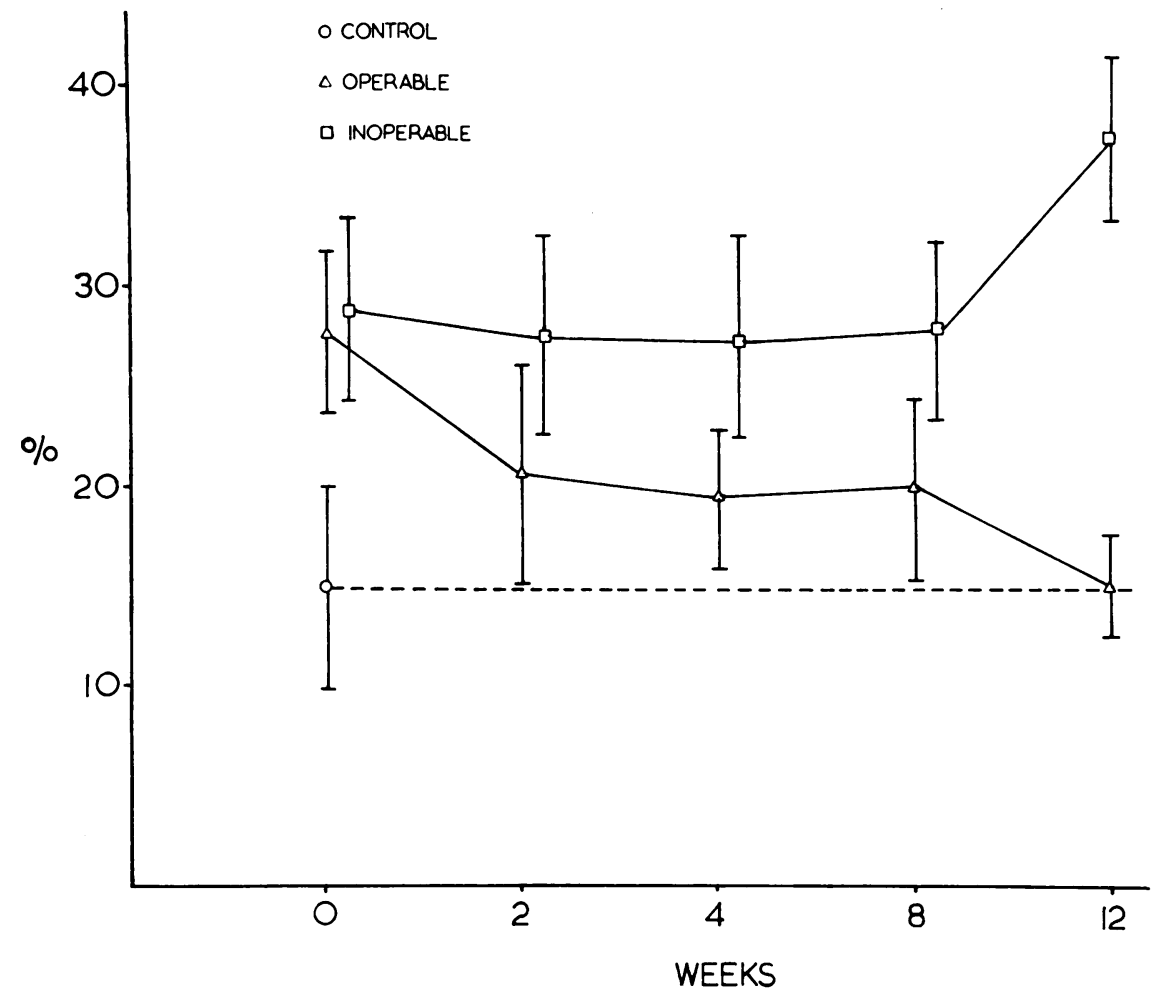

Fig. 2 Percentage depression of E-rosette formation by normal lymphocytes after incubation in serum from cancer patients.

carcinoma patients. Preoperatively the cancer sera was significantly $(P<0.001)$ more inhibitory than normal controls, and there was no difference in the inhibitory capacity of sera from operable and inoperable cancer. However, after the tumour had been removed the sera lost its inhibitory capacity and was no more inhibitory than normal sera at 12 weeks. The sera of the inoperable group, in contrast, became increasingly more inhibitory $(\mathrm{P}<0.001)$. The changes in the inhibitory property of sera correlated with E-rosette percentage, the coefficient of correlation being $0.73(\mathrm{P}<0.001)$.

\section{Discussion}

T-lymphocyte levels have shown a direct correlation with the clinical stage of a heterogeneous group of tumours (Babušikoa et al., 1975) and with carcinoma of the breast (Keller et al., 1976; Whitehead et al., 1976). Our findings of T-lymphocyte percentages in stomach and colorectal cancer support this observation. The T-lymphocyte level was depressed in all the cancer patients but the depression was significantly greater in the inoperable group of tumours, where the tumour load was greater.

In a heterogeneous group of tumours, total lymphocyte count alone was claimed to be an adequate measurement of cellular immune status, correlating with percentage E-rosette levels and absolute $\mathrm{T}$ and B cell counts (Harris et al., 1975). In our study, there is no difference in the absolute lymphocyte counts preoperatively, whereas the E-rosetting cell levels were depressed in the inoperable group. Such apparent differences may frequently be explained on grounds of methodology (Whitehead et al., 1978). In the post-operative period, the operable group of tumours shows no change in the total lymphocyte count in spite of a transient depression (at two weeks) and later recovery of E-rosette percentage and absolute T-lymphocyte count. In the inoperable group of tumours, however, there is a steady deterioration in total lymphocyte count correlating with the depression of percentage E-rosettes and absolute T-lymphocyte counts. Thus it appears that T-lymphocyte function as shown by E-rosetting is depressed early in the disease, the depression 
increasing with the tumour load, and does not correlate with total lymphocyte count, depression of which in advanced cases perhaps reflects the general debility of the patient.

Surgical trauma itself has been shown to depress PHA reactivity of lymphocytes in patients with benign surgical conditions (Riddle, 1967), probably because of its effect on the adrenal cortex. The same fact probably explains the finding of depressed E-rosettes and absolute $T$ cell counts one to two weeks after laparotomy in this study.

Most previous studies of the inhibitory effect of cancer sera on lymphocytes have used mitogen stimulation and some reports suggest that there is no effect of plasma taken from patients with solid tumours on allogenic lymphocytes taken from controls (Silk, 1967; Golob et al., 1969; Nelson, 1969), whereas other workers have demonstrated an inhibitory effect of cancer serum on healthy lymphocytes (McLaughlin and Brooks, 1974; Sample et al., 1971; Whittaker et al., 1971). We have previously shown that sera from breast cancer patients can ' inhibit E-rosette formation by normal T-lymphocytes (Whitehead et al., 1976, 1977). In this study we found significant inhibitory action of the serum from patients with carcinoma of the stomach, colon, or rectum on normal lymphocytes, as compared with the sera of healthy controls. Mild papain digestion of patients' lymphocytes removed this inhibitory effect of plasma and restored the E-rosetting to normal control levels. This finding demonstrated the presence of an E-rosette inhibitory factor in the serum of these patients. In the earlier study we showed in breast cancer that papain treatment of patients' lymphocytes would return percentage $T$ cell levels to normal but reincubation of lymphocytes in autologous sera inhibited the $T$ cell proportion again, showing the presence of an E-rosette inhibitory factor in the sera of breast cancer.

Twomey et al. (1974) demonstrated inhibitory action of serum on mitogen stimulation of normal lymphocytes in patients with head and neck cancer and this inhibitory action of serum disappeared in cured patients. Our findings also demonstrate a direct correlation between the tumour mass and the inhibitory action of serum on normal lymphocytes in that the inhibitory action of serum disappeared in those patients where the tumour was completely removed, but in inoperable tumours this inhibitory effect gradually increased in severity. The change in the inhibitory action of serum correlated with percentage E-rosette and absolute $\mathrm{T}$ cell count but not with total lymphocyte count. This finding gives additional support for the presence of E-receptor blocking factor in patients' serum which impairs the function of $T$ cells as determined by E-rosette forma- tion, but has no effect on their total numbers. This factor is related to tumour presence but its source and nature are not known. In an earlier study (Whitehead et al., 1977) we have demonstrated some evidence that this factor is a tumour disintegration product comprising cell debris or cell membrane fragments which bind to E-receptor sites of $\mathrm{T}$ lymphocytes.

The biological significance of this factor is considerable. It may be removed, at least temporarity, by plasmaphoresis or by infusion with proteolytic enzymes (Browne et al., 1976) leading to an improvement in the immune status. A continuing inhibitory effect of patients' serum after an apparent successful surgical removal would indicate residual disease, and the reappearance of this factor in a surgically cured patient would indicate recurrence, thus helping in the assessment of the patient; however, the effect of other forms of treatment - chemotherapy, radiotherapy, and immunotherapy where the main bulk of turnours remains in situ- on the presence of this serum factor is not known and requires investigation.

This work was carried out with the support of the Cancer Research Campaign. We are indebted to Mr. R. G. Newcombe for his statistical help and advice.

\section{References}

Al-Sarraf, M., Sardesai, S., and Vaitkevicius, V. K. (1971). Effect of syngeneic and allogeneic plasma on lymphocytes from cancer patients, patients with non-neoplastic diseases, and normal subjects. Cancer, 27, 1426-1432.

Anthony, H. M., Kirk, J. A., Madsen, K. E., Mason, M.K., and Templeman, G. H. (1975). E and EAC rosetting lymphocytes in patients with carcinoma of bronchus. 1 . Some parameters of the test and of its prognostic significance. Clinical and Experimental Immunology, 20, 29-40.

Babušíkova, O., Novotná, L., Korec, S., Schneková, K., Turková, D., Havranková, M., and Plentova, K. (1975). Changes in the proportion of $\mathrm{T}$ and $\mathrm{B}$ lymphocytes in human malignant neoplasia in relation to the clinical stage. Neoplasma, 22, 413-421.

Bolton, P. M., Mander, A. M., Davidson, J. M., James, S. L., Newcombe, R. G., and Hughes, L. E. (1975). Cellular immunity in cancer: Comparison of delayed hypersensitivity skin tests in three common cancers. British Medical Journal, 3, 18-20.

Browne, O., Bell, J., Holland, P. D. J., and Thornes, R. D. (1976). Plasmapheresis and immunostimulation (letter). Lancet, 2, 96.

Chapel, H. M. (1973). The effects of papain, trypsin and phospholipase A on rosette formation. Transplantation, 15, 320-325.

Golob, E. K., Israsena, T., Quatrale, A. C., and Becker, K. L. (1969). Effect of serum from cancer patients on homologous lymphocyte cultures. Cancer, 23, 306-308.

Harris, J. Stewart, T., Sengar, D. P. S., and Hyslop, D. (1975). Quantitation of T and B lymphocytes in peripheral blood of patients with solid tumours. 1. Relation to other parameters of in vivo and in vitro immune competence. Canadian Medical Association Journal, 112, 948-952.

Keller, S. E., Ioachim, H. L., Pearse, T., and Siletti, D.M. 
(1976). Decreased T-lymphocytes in patients with mammary cancer. American Journal of Clinical Pathology, 65, 445-449.

McLaughlin, A. P., and Brooks, J. D. (1974). A plasma factor inhibiting lymphocyte reactivity in urologic cancer patients. Journal of Urology, 112, 366-372.

Nelson, H. S. (1969). Delayed hypersensitivity in cancer patients: cutaneous and in vitro lymphocyte response to specific antigens. Journal of the National Cancer Institute. 42, 765-770.

Riddle, P. R. (1967). Disturbed immune reactions following surgery. British Journal of Surgery, 54, 882-886.

Sample, W. F., Gertner, H. R., Jr., and Chretien, P. B. (1971). Inhibition of phytohemagglutinin-induced in vitro lymphocyte transformation by serum from patients with carcinoma. Journal of the National Cancer Institute, 46, 1291-1297.

Silk, M. (1967). Effect of plasma from patients with carcinoma on in vitro lymphocyte transformation. Cancer, 20, 2088-2089.
Twomey, P. L., Catalona, W. J., and Chretien, P. B. (1974). Cellular immunity in cured cancer patients. Cancer, 33, 435-440.

Whitehead, R. H., Thatcher, J., Teasdale, C., Roberts, G.P., and Hughes, L. E. (1976.) $T$ and B lymphocytes in breast cancer. Stage relationship and abrogation of $T$ lymphocyte depression by enzyme treatment in vitro. Lancet, 1, 330-333.

Whitehead, R. H., Roberts, G. P., Thatcher, J., Teasdale, C., and Hughes, L. E. (1977). Masking of receptors for sheep erythrocytes on human T-lymphocytes by sera from breast cancer patients. Journal of the National Cancer Institute, 58, 1573-1576.

Whitehead, R. H., Roberts, G. P., Hughes, L. E., and Thatcher, J. (1978). Importance of methodology in demonstrating depression of T-lymphocyte levels. British Journal of Cancer, 37, 28-32.

Whittaker, M. G., Rees, K., and Clark, C. G. (1971). Reduced lymphocyte transformation in breast cancer. Lancet, 1, 892-893. 